\title{
THE
}

4-15-1987

\section{Unusual Critical Behavior in a Bilinear-Biquadratic Exchange Hamiltonian}

J. C. Bonner

University of Rhode Island

J. B. Parkinson

J. Oitmaa

H. W.J. Blöte

Follow this and additional works at: https://digitalcommons.uri.edu/phys_facpubs

Terms of Use

All rights reserved under copyright.

\section{Citation/Publisher Attribution}

J. C. Bonner, J. B. Parkinson, J. Oitmaa and H. W. J. Blöte. Unusual critical behavior in a bilinear-biquadratic exchange Hamiltonian. J. Appl. Phys. 61 (1987), 4432.

Available at: http://dx.doi.org/10.1063/1.338400

This Article is brought to you for free and open access by the Physics at DigitalCommons@URI. It has been accepted for inclusion in Physics Faculty Publications by an authorized administrator of DigitalCommons@URI. For more information, please contact digitalcommons-group@uri.edu. 


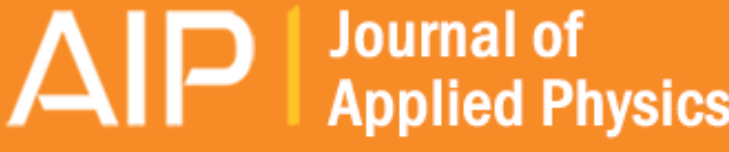

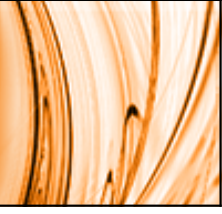

\section{Unusual critical behavior in a bilinear-biquadratic exchange Hamiltonian}

J. C. Bonner, J. B. Parkinson, J. Oitmaa, and H. W. J. Blöte

Citation: Journal of Applied Physics 61, 4432 (1987); doi: 10.1063/1.338400

View online: http://dx.doi.org/10.1063/1.338400

View Table of Contents: http://scitation.aip.org/content/aip/journal/jap/61/8?ver=pdfcov

Published by the AIP Publishing

\section{Articles you may be interested in}

The temperature dependence of the bilinear and biquadratic exchange coupling in $\mathrm{Fe} / \mathrm{Cu}, \mathrm{Ag} / \mathrm{Fe}(001)$ structures (abstract)

J. Appl. Phys. 75, 6473 (1994); 10.1063/1.356966

Spin-wave study of the magnetic excitations in a layered structure with bilinear and biquadratic interlayer exchange

J. Appl. Phys. 75, 6431 (1994); 10.1063/1.356952

Analysis of bilinear and biquadratic exchange coupling in $\mathrm{Fe} / \mathrm{Ag} / \mathrm{Fe}(001)$ trilayers

J. Appl. Phys. 73, 5966 (1993); 10.1063/1.353485

Application of the Green's function diagrammatic technique to complex crystal field systems with bilinear and biquadratic exchange

J. Appl. Phys. 49, 1419 (1978); 10.1063/1.324959

Biquadratic Exchange and Spin Exchange

AIP Conf. Proc. 10, 558 (1973); 10.1063/1.2946963

Frustrated by

old technology?

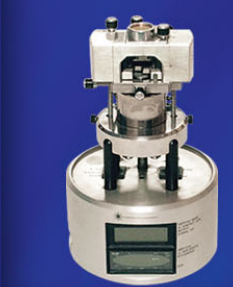

Is your AFM dead and can't be repaired?

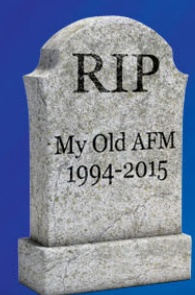

Sick of bad

customer support?

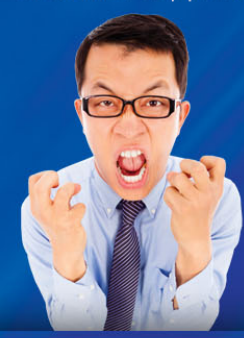

It is time to upgrade your AFM

Minimum \$20,000 trade-in discount for purchases before August 31st

Asylum Research is today's technology leader in AFM

dropmyoldAFM@oxinst.com 


\title{
Unusual critical behavior in a bilinear-biquadratic exchange Hamiltonian
}

\author{
J. C. Bonner \\ University of Rhode Island, Kingston, Rhode Island 02881-0817
}

J. B. Parkinson

University of Manchester Institute of Science and Technology (UMIST), Mancheste, United Kingdom

J. Oitmaa

University of New South Woles, Australia

H. W. J. Blöte

Technical University of Delft, Delft, The Netherlands

\begin{abstract}
We have performed a variety of numerical studies on the general bilinear-biquadratic spin-1 Hamiltonian $H / J=\Sigma_{i=1}^{N}\left[\mathbf{S}_{i} \cdot \mathbf{S}_{i+1}-\beta\left(\mathbf{S}_{i} \cdot \mathbf{S}_{i+1}\right)^{2}\right]$, over the range $0 \leqslant \beta \leqslant \infty$. The model is Bethe Ansatz integrable at the special point $\beta=1$, where the spectrum is gapless, but is otherwise believed to be nonintegrable. Affeck has predicted that an excitation gap opens up linearly in the vicinity of $\beta=1$. Our studies involving spectral excitations (dispersion spectra), scaled-gap, and finite-size scaling calculations are not consistent with the Affeck prediction. The situation appears complex, with novel crossover effects occurring in both regimes, $\beta<1$ and $\beta>1$, complicating the analysis.
\end{abstract}

A new way to investigate the critical properties of quantum spin chains of arbitrary spin $s$ has been proposed by Affeck, ${ }^{1,2}$ following on ideas of Haldane. ${ }^{3}$ Affeck has proposed a number of mappings between varions types of spin chain and two-dimensional (2D) continuum field theories, namely nonlinear $\sigma$ models and related Wess-Zumino modeis. He extends the classification of critical theories in $2 \mathrm{D}$ by constraints of conformal invariance on systems with continuous symmetries. Conformal invariance in these Wess-Zumino models allows computation of the anomalous dimension of operators from which the critical exponents of the quantum spin chains are obtained. Numerical verification (or otherwise) of the various Affeck mappings by a detailed study of the particular spin chains is both timely and important.

The properties of quantum spin chains of higher spin are of interest from a different point of view. Stimulated by a prediction of Haldane, a variety of studies has appeared on the spin-1 Heisenberg antiferromagnet (AFM) generalized by the addition of $X X Z$ spir anisotropy and also single-ion anisotropy. The resulting apparent rich and complicated phase diagram has come as a surprise, since the Heisenberg spin chains may be reasonably regarded as the simplest magnetic systems, previously thought to be generically similar for all $s$. Haldane predicts that the class of integer-spin AFM $X X Z$ spin chains shows radically different $T_{c}=0$ critical behavior from the class of half-integer-spin chains which should resemble the exactly solved spin- $1 / 2 X X Z$ model. We are interested in pursuing a rather different point of view: $X X Z$ spin chains for $s>1 / 2$ are believed to be nonintegrable, and hence the integrable spin-1/2 chain is special. We are therefore, investigating possible nonintegrability effects in quantum chains of higher spin. ${ }^{4}$

This paper reports investigations of the excitation spectra and energy gaps of the general 10 spir-1 bilinear-biquadratic exchange Hamiltonian

$$
\frac{H}{J}=\sum_{i=1}^{N} \mathbf{S}_{i} \cdot \mathbf{S}_{i+1}-\beta \sum_{i=1}^{N}\left(\mathbf{S}_{i} \cdot \mathbf{S}_{i+1}\right)^{2}
$$

$\beta \geqslant 0$, by analytic studies and by numerical calculations up to $N=12$ spins. This Hamiltonian is believed to be nonintegrable except at the special point $\beta=1$. Hamiltonian (1) for $\beta=1$ in fact is the spin-1 version of an integrable class of modeis with general spin-s and SU (2) symmetry. ${ }^{5,6}$ They all show a qualitative resemblance to the spin-1/2 member of the family, which is the familiar spin-1/2 Heisenberg AFM chain. Special limits of the model are therefore

(1) $\beta=1$ : integrable "Russian" model;

(2) $\beta=0$ : nonintegrable Heisenberg modei;

(3) $\beta=\infty$ : nonintegrable pure biquadratic model.

The model is gapless with continuous symmetry at the Russian point $\beta=1$, whereas the Haldane prediction, supported by numerical results, is that a gap is present in the Heisenberg limit, $\beta=0 .{ }^{8}$ The question of interest is therefore where the gap disappears, and the value of the critical exponent governing the disappearance of the gap. Affleck predicts that the system is gapless only at $\beta=1$, and that an excitation gap opens up linearly in the vicinity of this point, ${ }^{1}{ }^{1}$ i.e.,

$$
\Delta E / J \sim|1-\beta| \text {. }
$$

Studies have recently been performed on this system. ${ }^{9-11}$ The focus of Ref. 10 has been a survey of the two regimes, $\beta<1$ and $\beta>1$, in terms of the excitation spectra as a function of the wave-vector $k$ and $S_{T}^{z}$, and also scaled-gap studies of the finite $N$ singlet-triplet excitation gap. The focus of Ref. 11 has been a detailed finite-size scaling study of Hamiltonian (1) aimed principally at finding the gap exponent, and the conclusions are qualitatively in agreement with Affleck, but quantitatively the singlet-triplet gap opens up more slowly than predicted, behaving to a close approximation as

$$
\Delta E / J \sim|1-\beta|^{5.8} .
$$

The slow opening of the gap as found in Ref. 11 is consistent with the results of Ref. 10, where scaled-gap calculations predict that the gap might vanish in the $\beta<1$ regime for $\beta \geqslant 0.6$, i.e., if a gap exists for $0.6<\beta<1$ it is very small. The 


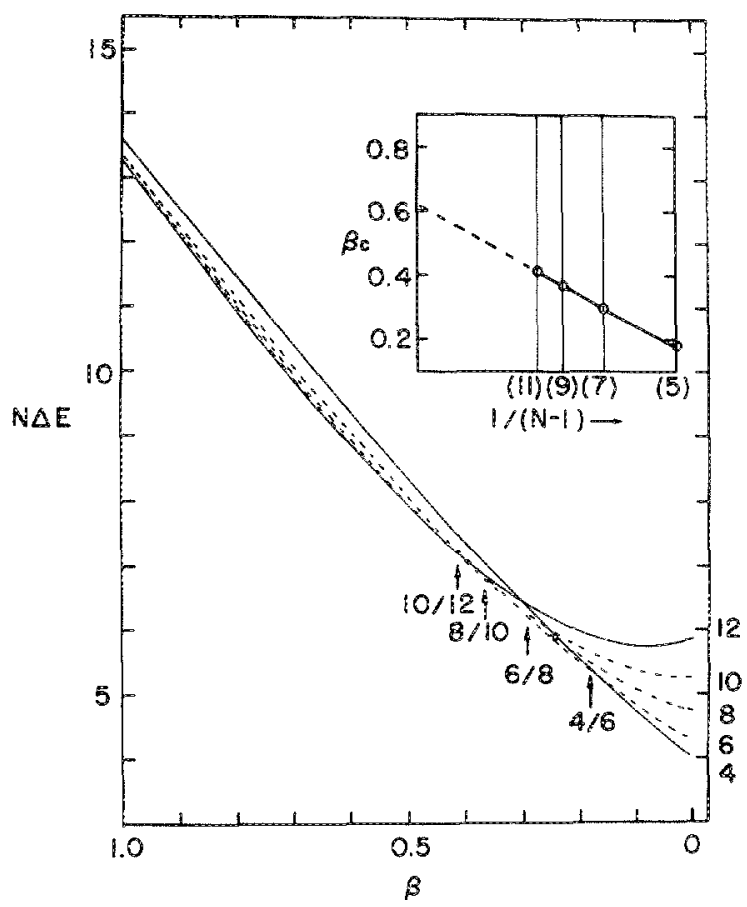

FIG. 1. Scaled-gap plots for the range $0 \leqslant \beta<1$, indicating successive crossing points $\beta_{c}^{(N, N-2)}$ located by the arrows. A rough plot of the $\beta_{c}^{(N, N-2)}$ vs $(N-1)^{-1}$ shown as the inset indicates a limiting value $\beta_{c} \leq 0.6$. The system has a gap for $0 \leqslant \beta<\beta_{\mathrm{c}}$.

scaled-gap caiculations, shown in Fig. 1, are supported by a study of dispersion spectra in a field, since a crossover in the lowest-lying excitations from gap to gapless character may be expected to correlate with crossover effects in the entire spectrum. A study of the lowest-lying excitations of the spin1 Heisenberg AFM as a function of the wave-vector $k$ and magnetic field has revealed an interesting crossover effect. ${ }^{12}$ It appears that half the dispersion spectra, corresponding to large $S_{T}^{z}$ values (high fields) show a qualitative resemblance to the entire set of dispersion curves for the spin-1/2 Heisenberg AFM. In particular, a soft mode tracks across the Brillouin zone as the magnetic field varies between $\sim 1 / 2 H_{s}$ and $H_{s}$ where $H_{s}$ is the saturation critical value. This regime corresponds to $S_{T}^{z}=N, N-1, \ldots, N / 2$. The other half of the dispersion spectra corresponds to $S_{T}^{z}=N / 2, N / 2-1, \ldots, 0$ (low fields). No tracking soft mode appears, and the curves show a striking resembiance to classical dispersion spectra in a field.

Correspondingly, an analytic and numerical study has been performed of the field-cependent dispersion spectra for $N$ finite as well as $N \rightarrow \infty$ for the Russian Hamiltonian. ${ }^{13}$ Again the excitations divide into two sets, but the two sets are now specified by dispersion curves with $S_{T}^{z}$, even on the one hand, and $S_{T}^{z}$ odd on the other. The $S_{T}^{z}$ odd excitation set shows more finite-size distortion for finite $N$, but analytic considerations determine that the two sets become degenerate in the thermodynamic limit. ${ }^{13}$ Hence it is clear that the field-dependent excitation patterns are strikingly different in the two limits, and a crossover must occur in the region $0 \leqslant \beta \leqslant 1$. Analytic considerations lead us to term the $S_{T}^{z}$ even/odd excitation pattern characteristic of the $\beta=1$ limit the "two-string" picture, ${ }^{13}$ and we denote the high $S_{T}^{z} /$ low $S_{T}^{z}$ excitation pattern characteristic of the $\beta=0$ limit as the "one-string" or "single-deviation" picture. The question is whether the crossover is sharp or spread over a range of $\beta$. Analytic considerations based on the always integrable twospin deviation problem ${ }^{14}$ suggest a sharp crossover at $\beta$ $=1 / 3$. This would be a reasonable expectation if Hamiltonian (1) were integrable. In fact, we find numerically that there appears to be a $\beta$ region, whose extent increases with increasing $N$, where the excitations are "confused" over which picture best describes them. This region extends over at least the range $1 / 3<\beta \geqslant 0.6$ and probably over the range $1 / 3<\beta<1$. This "smeared" crossover region is consistent with ideas on nonintegrability and quantum chaos. ${ }^{4,15}$

We have found complexities associated also with the regime $\beta>1$. Scaled-gap calculations ${ }^{10}$ and a finite-size scaling analysis " both indicate that a singlet-triplet excitation gap opens up for $\beta<1$. However, it was subsequently observed $^{10}$ that for $N \geqslant 8$ at the biquadratic limit the lowest excited state was no longer the triplet state at $k=\pi$ (with singlet ground state at $k=0$ ) but a secona singlet at $\pi$. Subsequent studies in the range $\beta>1$ show that the singlet at $\pi$ crosses below the tripiet at $\pi$ to become the dominant lowlying excited state at $\beta^{-1} \sim 0.097$ for $N=8, \beta^{-1} \sim 0.231$ for $N=10$, and $\beta^{-1} \sim 0.334$ for $N=12$ as illustrated in Fig. 2. A plot of these values versus $N^{-1}$, shown as Fig. 3, is almost linear, extrapolating to $\beta^{-1} \sim 0.85$ as $N \rightarrow \infty$, a value which could well be $\beta=1$. Evidently an interesting, and heretofore unobserved, crossover phenomenon in the context of finitesize scaling analysis, is present. Furthermore, direct extrapolations versus $N^{-1}$ of the following excitation gaps: (a) sing-

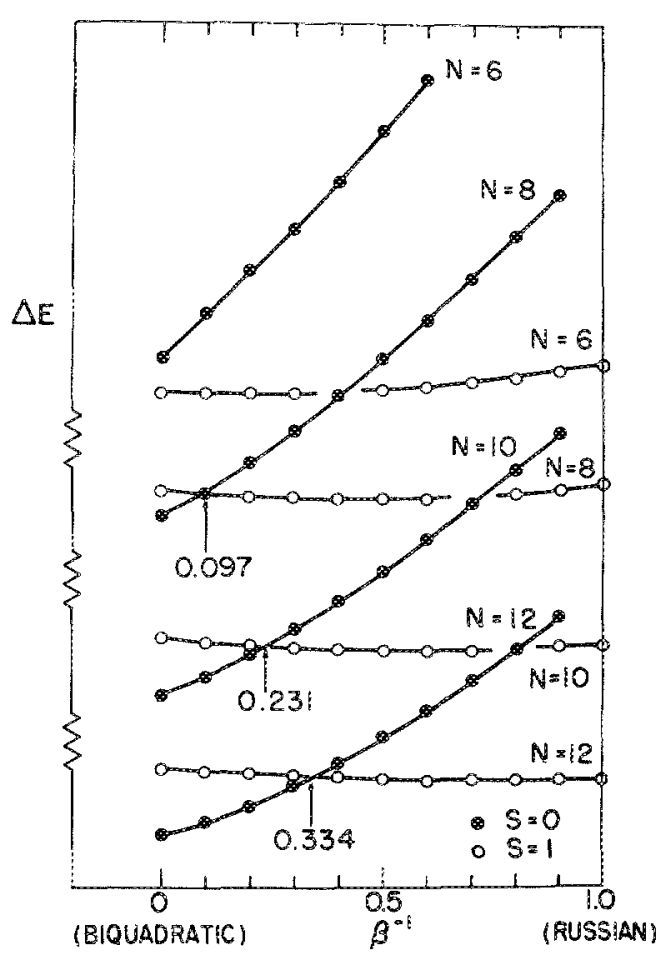

FIG. 2. Comparative plot of singlet and rniplet excitations as a function of $B$ '. For $N=6$, the triplets always lie lower. For $N>6$, the excitations cross at a $\beta^{-1}$ value which increases with $N$. 


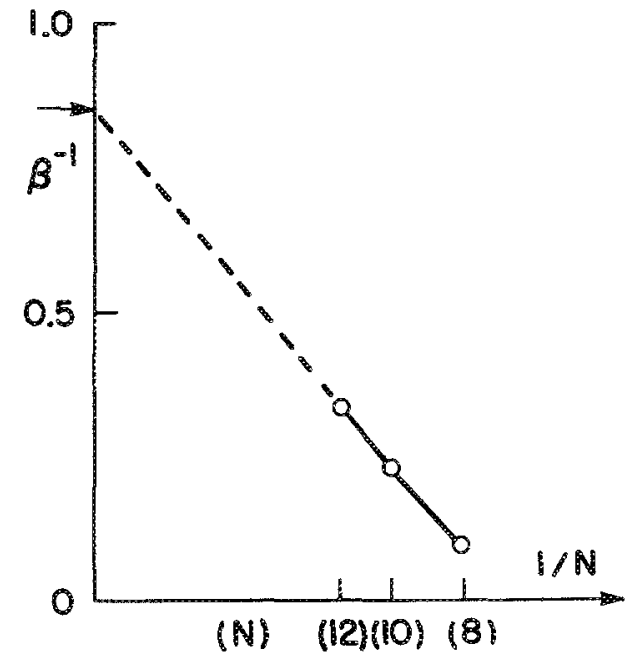

FIG. 3. A plot of the values of $\beta^{-1}$ at which the lowest excited state in the range $0 \leqslant \beta^{-1} \leqslant 1$ changes from singlet to triplet vs $N^{-1}$. The rough extrapolated value is consistent with the value unity, implying that the singlet is ultimately the dominant excited state in this range.

let at $\pi,(\mathrm{b})$ triplet at $\pi$, (c) quintet at $k=0$, and (d) singlet at $k=0$ over the $\beta$ range between Russian and biquadratic, predict either no gap in this range (singlet at $\pi$ ) or a very small gap of $\Delta E / J<0.1$ [compare with the value $\Delta E /$
$J \sim 0.41$ (Ref, 16) for the Haldane gap]. These results suggest the regime $1 \leqslant \beta \leqslant \infty$ could be gapless, in contrast to the predictions of finite-size scaling and Afleck. ${ }^{1}$ This anomalous situation is undergoing further investigation.

We are happy to acknowledge useful discussions with G. Müller and M. P. Nightingale. This work was supported (for J.C.B.) by NSF grant DMR86-03036 and the SERC (UK); by a grant from CSRO (J.O.); by NATO and by the ZWO (Netherlands) (H.W. J. B.).

1. Affleck, Nucl. Phys. B 265, 409 (1986).

${ }^{21}$. Affleck, Phys. Rev. Lett. 56, 746 (1986)

${ }^{3}$ F. D. M. Haldane, Phys. Lett. 93A, 463 (1983); Phys. Rev. Lett. 50, 1153 (1983)

${ }^{4}$ G. Müller, J. C. Bonner, and J. B. Parkinson (these proceedings),

${ }^{5}$ L. A. Takhtajan, Phys. Lett. 87A, 479 (1982).

${ }^{6}$ H. M. Babujian, Phys. Lett. 90A, 479 (1982).

7 We term the model "Russian" for convenience in the light of Refs. 5 and 6. ${ }^{8} \mathrm{~J}$. C. Bonner (these proceedings).

${ }^{\circ} \mathrm{D}$. Kung (unpublished work).

10J. Oitmaa, J. B. Parkinson, and J. C. Bonner, J. Phys, C 19, L595 (1986).

${ }^{\prime H}$. W. J. Blöte and H. Capel, Physica A (in press).

12J. B. Parkinson and J. C. Bonner, Phys. Rev. B 32, 4703 (1985).

${ }^{13}$ J. B. Parkinson and J. C. Bonner, J. Phys. C 19, 6063 (1986) and unpublished work.

${ }^{14}$ R. P. Hodgson and J. B. Parkinson, J. Phys. C 18, 6385 (1985).

${ }^{15} \mathrm{G}$. Müller, Phys. Rev. A 34, 3345 (1986).

${ }^{15}$ M. P. Nightingale and H. W. J. Blöte, Phys. Rev. B 33, 659 (1986). 